\title{
Presencia del genotipo Beijing entre cepas del complejo Mycobacterium tuberculosis en dos centros de salud de la Región Metropolitana-Chile
}

\author{
Paulina Meza, M. Elvira Balcells, Carolina Miranda, Marcela Cifuentes, Aniela Wozniak y Patricia García
}

\section{Presence of Bejing genotype among Mycobacterium tuberculosis strains in two centres of the Region Metropolitana of Chile}

Background: Genotyping of Mycobacterium tuberculosis complex (cMtb) allows us to know geographically predominant lineages. Some lineages spread more rapidly and are associated with multidrug resistance, particularly Beijing, which has been reported in Latin America (Peru). There is little information about this topic in Chile and there are no reports of the presence of the Beijing genotype. Aim: To determine the most prevalent lineages in the Metropolitan Region of Chile with emphasis on the search for Beijing in two health centers. Methods: Two complementary molecular methods were used: spoligotyping, based on the variations of the direct repeat regions in the genome of cMtb and MIRU-VNTR, based in the variable number of tandem repeats of mycobacterial interspersed repetitive units, and subsequent analysis in international databases. A designed lineage was assigned to 37 of the 43 strains studied (86\%); 6 isolates could not be assigned to any genotype. LAM and T genotype were the most frequent (39.5 and 32.5\%, respectively) followed by Haarlem (7.0\%), Beijing (4.7\%) and X (2.3\%). Conclusion: We describe for the first time the presence of the Beijing genotype in Chile. cMtb molecular surveillance should be implemented in our country in order to know the dynamics of its transmission.

Key words: Molecular epidemiology, Mycobacterium tuberculosis complex, genotypes, MIRU-VNTR, spoligotyping, Beijing.

Palabras clave: Epidemiologia molecular, complejo Mycobacterium tuberculosis, genotipos, MIRU-VNTR, espoligotipificación, Beijing.

\section{Introducción}

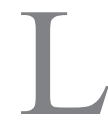

a epidemiología molecular de la tuberculosis (TBC) ha aportado información de gran utilidad respecto de la transmisión de la enfermedad, utilizándose como un complemento a la epidemiología clásica. El conocimiento de los distintos genotipos del complejo Mycobacterium tuberculosis (cMtb) ha permitido conocer los tipos más prevalentes en determinadas localizaciones geográficas ${ }^{1}$. Hoy se sabe que ciertos genotipos se diseminan con mayor rapidez (mayor transmisibilidad) o que causan enfermedad de mayor gravedad, asociado a la multi-resistencia; también se ha establecido relaciones entre los genotipos y la respuesta inmunológica del hospedero $^{2,3}$ lo que justifica la necesidad de identificar aquellos prevalentes en cada país o región. Se han descrito como prevalentes en el mundo los genotipos Haarlem, Latino América-Mediterráneo, T, Este de África-India, Central-Asia, X y Beijing. El genotipo Haarlem (H), descrito en el año $1999^{4}$ en pacientes nederlandeses, es prevalente en los Países Bajos y el norte de África donde ha causado brotes de tuberculosis multi-resistente. El genotipo Latino América-Mediterráneo (LAM), el prevalente en Latinoamérica y España, sugiriendo orígenes comunes relacionado a la colonización hispana ${ }^{1,5,6}$. El genotipo $T$, mal definido y localizado en países de varios continentes como Etiopía, Rusia, Madrid y Europa Central ${ }^{1}$. El genotipo Este de África-India (EAI), muy frecuente en áreas del lejano Oriente, Asia y Oceanía ${ }^{1,7}$. El genotipo Central-Asia (CAS) o Delhi, localizado en el Medio Oriente, Asia Central, Europa y Australia, lo que lo vincula con los inmigrantes del sur de Asia ${ }^{1}$. El genotipo $X$, prevalente en América del Norte y regiones de América Central sugiriendo una ascendencia anglosajona o incluso afro-americana ${ }^{1,6}$ y por último el genotipo Beijing, endémico y con un claro predominio en ciertas regiones del extremo Oriente, Asia y Oceanía, exceptuando India subcontinental y Sudáfrica ${ }^{1,8,9}$, se ha asociado a alta transmisibilidad, multi-resistencia ${ }^{8,10}$ y mayor poder patógeno asociado a la baja inducción de citoquinas inflamatorias, lo que permitiría un mayor crecimiento intracelular del $\mathrm{cMtb}^{2,11}$. En los Estados Unidos de América, específicamente en Nueva York, en el año 1991, durante un brote que afectó a ocho pacientes
Pontificia Universidad Católica de Chile.

Servicio de Laboratorios Clínicos Laboratorio de Microbiología. (PM, CM).

Escuela de Medicina Departamento de Enfermedades Infecciosas (EB).

Departamento de Laboratorios Clínicos (AW, PG).

Hospital San Borja Arriarán, Santiago.

Laboratorio de Microbiología (MC)

Proyecto financiado por Fondos Departamentales Concursables del Departamento de Laboratorios Clínicos. Este Proyecto es parte de una tesis para optar al grado académico de Magister en Ciencias Biomédicas otorgado por la Universidad de Talca (P.M.A.)

Los autores declaran no tener conflictos de interés.

Recibido: 16 de mayo de 2013 Aceptado: 24 de noviembre de 2013

Correspondencia a: Patricia García Cañete pgarcia@med.puc.cl 
con TBC resistente a isoniacida, rifampicina, etambutol, estreptomicina, kanamicina, etionamida y rifabutina, se identificó el genotipo "W", que fue posteriormente asociado a otros cinco brotes en hospitales del área de Nueva York. Este genotipo se relacionó estrechamente con el genotipo Beijing (Beijing W) ${ }^{12,13}$. En América Latina, a pesar del bajo reporte de genotipo Beijing, éste ha sido detectado en forma significativa en cepas peruanas, tanto susceptibles como resistentes, lo cual podría explicarse por la migración asiática ${ }^{6,8}$.

En Chile existe muy poca información sobre los genotipos predominantes del cMtb. En el año 2006, Mancilla y cols., reportaron las variantes genéticas (espoligotipos) de M. tuberculosis en la Región de Los Lagos, en cepas de 25 pacientes con TBC, no encontrándose el espoligotipo (ST) correspondientes al genotipo Beijing ${ }^{14}$. En el año 2008 y posteriormente en el año 2012, Ritacco y cols., en Buenos Aires, Argentina, estudiaron cepas de América Latina, incluyendo 35 y 13 cepas de Chile, respectivamente. Estos estudios no caracterizaron la totalidad de los cepas y sólo se describe el genotipo LAM en una cepa, sin encontrar el genotipo Beijing ${ }^{5,13}$.

El objetivo de este trabajo fue conocer los genotipos prevalentes en dos centros de salud de la Región Metropolitana de Santiago de Chile con énfasis en la búsqueda del genotipo Beijing.

\section{Material y Método}

\section{Obtención y caracterización de cepas del complejo M. tuberculosis}

Se estudió inicialmente un total 107 cultivos positivos, sugerentes de cMtb, obtenidos entre los años 2008 y 2011 en forma consecutiva, procedentes de pacientes de la Red Salud UC y del Hospital Clínico San Borja-Arriarán. Los cultivos fueron mantenidos en los laboratorios de $\mathrm{Mi}$ crobiología de ambos centros en medio sólido o líquido. La identificación presuntiva fue realizada de acuerdo a características macroscópicas de las colonias. Los 107 cultivos fueron resembrados en medio sólido Lowenstein Jensen y se recuperaron solamente 47 cepas, de las cuales, 37 provenían de pacientes de la Red Salud UC y 10 de pacientes del Hospital Clínico San Borja-Arriarán. Se realizó la búsqueda en el Sistema Informático (SIL) de datos como edad, sexo y la comuna de los pacientes. Las 47 cepas estudiadas provenían de 44 pacientes, es decir, en 3 pacientes se incluyeron 2 cepas. De los 44 pacientes, $52 \%$ eran de sexo femenino; $64 \%$ de las muestras fueron obtenidas del tracto respiratorio, mayoritariamente muestras de expectoración. La comuna de residencia de los pacientes se distribuyó de la siguiente manera: $39 \%$ pertenecían a la comuna de Maipú, $25 \%$ a Santiago Centro y en $20 \%$ no se obtuvo el dato de residencia.

\section{Extracción de ADN e identificación molecular del cMtb}

Se tomaron entre una y cinco colonias sugerentes de cMtb y se emulsionaron en $300 \mu \mathrm{l}$ de agua destilada para posteriormente realizar la inactivación con calor (20 min a $95^{\circ} \mathrm{C}$ ) y lisado con sonicador $(15 \mathrm{~min})$. Luego se centrifugó 3 min a $13.000 \mathrm{rpm}$ para obtener el sobrenadante que contiene el ADN, el que se almacenó a $-20^{\circ} \mathrm{C}$ hasta su posterior uso. La identificación de las 47 cepas fue confirmada con una RPC convencional utilizando partidores que amplifican un fragmento de 1020 pares de bases del gen de la girasa B ( $g y r B)$, específico para cMtb, según el protocolo descrito por Kasai y cols. ${ }^{15}$.

Los partidores utilizados fueron MTUB f: 5'-TCGGACGCGTATGCGATATC y MTUB r: 5'ACATACAGTTCGGACTTGCG. Cada reacción de amplificación por RPC contenía: $12,5 \mu \mathrm{l}$ premezcla $2 \mathrm{X}, 2 \mu \mathrm{l}$ de cada partidor (forward y reverso) $10 \mu \mathrm{M}, 0,2 \mu \mathrm{l}$ de ADN polimerasa GoTaq-flexi (Promega ${ }^{\circledR}$ ) $5 U / \mu 1,5 \mu l$ de agua destilada libre de nucleasas y $5 \mu \mathrm{l}$ de ADN de las muestras o controles (control negativo con agua destilada; control positivo con ADN de cMtb), con un volumen final de $25 \mu 1$. El programa de amplificación fue del tipo touch-down que consiste en una denaturación inicial de 3 min a $95^{\circ} \mathrm{C}$ y 10 ciclos sucesivos en los cuales la denaturación es de $30 \mathrm{~s}$ a $95^{\circ} \mathrm{C}$ y la extensión de $30 \mathrm{~s}$ a $72^{\circ} \mathrm{C}$. El annealing es de $30 \mathrm{~s}$ a $65^{\circ} \mathrm{C}$ para el primero de los 10 ciclos y cada ciclo sucesivo disminuye un ${ }^{\circ} \mathrm{C}$ hasta llegar a $56^{\circ} \mathrm{C}$. Posteriormente ocurren 35 ciclos iguales de $30 \mathrm{~s}$ a $95^{\circ} \mathrm{C}, 30 \mathrm{~s}$ a $55^{\circ} \mathrm{C}$ y $30 \mathrm{~s}$ a $72^{\circ} \mathrm{C}$. Por último, ocurre una extensión final de $10 \mathrm{~min}$ a $72^{\circ} \mathrm{C}$. Los productos amplificados se analizaron mediante electroforesis en geles de agarosa al 1,5\% teñidos con bromuro de etidio, en tampón TAE y la visualización se realizó en transiluminador con luz UV.

\section{Genotipificación por MIRU-VNTRs}

La genotipificación por MIRU-VNTR se realizó mediante una RPC convencional, amplificando 12 de los 41 loci: 02, 04, 10, 16, 20, 23, 24, 26, 27, 31, 39, 40. Las RPC se realizaron con los partidores descritos por Cowan y cols. ${ }^{16}$. La mezcla que se utilizó en la amplificación por RPC contenía: $12,5 \mu 1$ premezcla $2 \mathrm{X}, 2,5 \mu \mathrm{l}$ de cada partidor (forward y reverso) $5 \mu \mathrm{M}, 0,2 \mu \mathrm{l}$ de la GoTaq flexi ADN polimerasa $5 \mathrm{U} / \mu \mathrm{l}$ (Promega $\left.{ }^{\circledR}\right), 5,3 \mu \mathrm{l}$ de agua destilada libre de nucleasas y $2 \mu \mathrm{lde}$ ADN de las muestras o controles, con un volumen final de $25 \mu \mathrm{l}$. El programa de amplificación fue del tipo touch-down según lo descrito anteriormente. La visualización de los productos de amplificación se realizó mediante electroforesis en geles de agarosa al 1,5\%-2\% (según el tamaño esperado) teñidos con bromuro de etidio, en tampón TAE junto con un estándar de tamaño de 50 pb. Se calculó el número de repeticiones presentes para cada MIRU, en cada cepa de 


\section{Microbiología}

acuerdo al tamaño del producto amplificado obtenido, según lo descrito por Cowan y cols. ${ }^{16}$.

\section{Genotipificación por espoligotipificación}

Se realizó con el kit comercial Ocimum Biosolutions $\mathrm{BV}{ }^{\circledR}$, India, de acuerdo a las instrucciones del fabricante $^{17,18}$. Este kit contiene partidores Dra y Drb que amplifican las secuencias conservadas de la región DR, controles positivos, membrana con oligos complementarios a cada uno de los 43 interespaciadores. Primero se realizó una RPC y los reactivos para la mezcla $1 \mathrm{X}$ fueron: $4 \mu \mathrm{l}$ de partidores Dra y Drb $(20$ pmol $), 4 \mu \mathrm{l}$ dNTP MIX (2,5 mM de cada nucleótido), $5 \mu$ lampón Tth (10x), 0,1 $\mu \mathrm{l}$ Tth polimerasa ( 5 unidades $/ \mu \mathrm{l}), 4 \mu \mathrm{lde}$ $\mathrm{MgCL}_{2}(25 \mathrm{mM}), 27 \mu \mathrm{l}$ de agua libre de nucleasas y $2 \mu \mathrm{l}$ de ADN de cada una de las cepas a estudiar. Se utilizaron dos controles positivos: M. tuberculosis H37Rv y $M$. bovis BCG P3 y un control negativo (agua destilada). El programa de amplificación utilizado consistió en denaturación inicial a $96^{\circ} \mathrm{C}$ por $3 \mathrm{~min}$, seguido de 20 ciclos de denaturación a $96^{\circ} \mathrm{C}$ por $1 \mathrm{~min}$, annealing a $55^{\circ} \mathrm{C}$ por $1 \mathrm{~min}$ y extensión a $72^{\circ} \mathrm{C}$ por $30 \mathrm{seg}, \mathrm{y}$ con una extensión final a $72^{\circ} \mathrm{C}$ por $5 \mathrm{~min}$. El producto amplificado fue denaturado a $99{ }^{\circ} \mathrm{C}$ por $10 \mathrm{~min}$ y a continuación hibridado contra la membrana utilizando el soporte Miniblotter MN 45. La membrana contenía los 43 oligonucleótidos complementarios, fijados covalentemente, derivados de la región espaciadora de las DR de las cepas de M. bovis P3 y M. tuberculosis H37Rv. Luego de una incubación con calor y posteriores lavados se incubó la membrana hibridada con un conjugado de estreptavidina- peroxidasa (Roche) para luego realizar una reacción de quimioluminiscencia con el sistema ECL (Amersham) y finalmente fue revelado con sistema fotográfico. La determinación de la presencia o ausencia del espaciador se realizó visualmente. Los patrones observados se registraron en formato binario para el posterior análisis de la relación filogenética entre las cepas en las bases de datos (miru-vntrplus y del Instituto Pasteur-Guadeloupe).

\section{Bases de datos}

Se ingresaron los resultados de genotipificación por MIRU-VNTR y espoligotipificación a las bases de datos SITVITWEB para obtener MIRU International Type (MIT) y Spoligotyping International Type (SIT), respectivamente (URL: http://www.pasteur-guadeloupe. fr:8081/SITVIT_ONLINE/ $/)^{19}$. Para obtener genotipos se ingresaron a la base de datos MIRU-VNTRplus (URL: http.//www.miru-vntrplus.org) $)^{20,21}$.

El estudio fue presentado y aprobado por el Comité de Ética de la Investigación de la Escuela de Medicina de la Pontificia Universidad Católica de Chile (CEI-UC) con el número de proyecto 12-016.

\section{Resultados}

De las 47 cepas estudiadas, 43 fueron positivas en la amplificación del gen $g y r B$, confirmando que pertenecen al cMtb, las que finalmente fueron consideradas en los resultados.

\section{Análisis de MIRU-VNTR}

Se obtuvo productos de amplificación para las 43 cepas en los 12 tipos de MIRU estudiados, mediante 12 RPC para cada cepa. La frecuencia del número de repeticiones para cada tipo de MIRU, para todas las cepas estudiadas, se muestra en la Tabla 1. En algunas cepas para los MIRU31 y MIRU40, se obtuvo un tamaño del producto amplificado menor que lo descrito para una repetición, por lo que el dígito asignado fue cero. El número de repeticiones resultantes permite obtener el patrón de 12 dígitos, en el que cada número representa el número de repeticiones para cada tipo de MIRU. La introducción del patrón de 12 dígitos en la base de datos SITVITWEB permite obtener el número MIT (MINU international type). Estos resultados se muestran en la Tabla 2. Se obtuvo un resultado de MIT para 25 de las 43 cepas estudiadas (58\%). Los 18 restantes, corresponden a series MIT no definidas en la base de datos (huérfanas). De los 25 MIT obtenidos, 19 son diferentes entre sí y 6 corresponden a dos grupos de 2 y 4 MIT idénticos entre sí, siendo este último, MIT 190 (patrón 124326153220), la agrupación más frecuente.

\section{Análisis de espoligotipo}

El análisis de las 43 cepas mostró que había 25 patrones (espoligotipos) diferentes. La introducción de los

\begin{tabular}{|c|c|c|c|c|c|c|c|c|c|c|}
\hline \multirow[b]{2}{*}{ MIRU } & \multicolumn{10}{|c|}{ Número de repeticiones (\%) } \\
\hline & 0 & 1 & 2 & 3 & 4 & & 5 & 6 & 7 & 8 \\
\hline 02 & & $9(20,9)$ & $34(79,1)$ & - & - & & - & - & - & - \\
\hline 04 & - & $1(2,3)$ & $42(97,7)$ & - & - & & - & - & - & - \\
\hline 10 & - & - & $1(2,3)$ & $13(30,3)$ & $28(65,1)$ & & - & $1(2,3)$ & -- & \\
\hline 16 & - & $1(2,3)$ & $9(20,9)$ & $30(69,8)$ & $3(7,0)$ & & - & - & - & - \\
\hline 20 & - & $4(9,3)$ & $39(90,7)$ & - & - & & - & - & - & - \\
\hline 23 & - & $2(4,7)$ & - & - & - & 20( & $(46,5)$ & $20(46,5)$ & - & $1(2,3)$ \\
\hline 24 & - & $43(100)$ & - & - & - & & - & - & - & - \\
\hline 26 & - & - & - & $8(18,6)$ & $8(18,6)$ & 21 & $(48,8)$ & $3(7,0)$ & $3(7,0)$ & - \\
\hline 27 & - & $1 \quad(2,3)$ & $1 \quad(2,3)$ & $39(90,7)$ & $2 \quad(4,7)$ & & - & - & - & - \\
\hline 31 & $1 \quad(2,3)$ & - & $17(39,6)$ & $24(55,8)$ & - & 1 & $(2,3)$ & - & - & - \\
\hline 39 & - & - & $41(95,3)$ & $2(4,7)$ & - & & - & - & - & - \\
\hline 40 & $6(14,0)$ & $1 \quad(2,3)$ & $5(11,6)$ & $12(27,9)$ & $9(20,9)$ & 3 & $(7,0)$ & $7(16,3)$ & - & - \\
\hline
\end{tabular}


Tabla 2. Número de repeticiones (MIRU-VNTR) y MIT para las cepas estudiadas

\begin{tabular}{|c|c|c|c|c|c|c|c|c|c|c|c|c|c|}
\hline \multirow{2}{*}{$\begin{array}{l}\mathrm{n} \text { de } \\
\text { cepas }\end{array}$} & \multicolumn{12}{|c|}{ MIRU } & \multirow[t]{2}{*}{ MIT* } \\
\hline & 02 & 04 & 10 & 16 & 20 & 23 & 24 & 26 & 27 & 31 & 39 & 40 & \\
\hline 37 & 2 & 2 & 3 & 4 & 2 & 5 & 1 & 3 & 3 & 3 & 2 & 2 & 340 \\
\hline 39 & 2 & 2 & 4 & 3 & 1 & 5 & 1 & 5 & 3 & 3 & 2 & 3 & 27 \\
\hline 42 & 2 & 2 & 4 & 2 & 1 & 6 & 1 & 4 & 3 & 2 & 2 & 0 & huérfana \\
\hline 43 & 2 & 2 & 4 & 2 & 1 & 6 & 1 & 4 & 3 & 2 & 2 & 0 & huérfana \\
\hline 47 & 2 & 2 & 3 & 4 & 2 & 5 & 1 & 5 & 3 & 3 & 2 & 2 & 157 \\
\hline 50 & 2 & 2 & 4 & 3 & 2 & 6 & 1 & 5 & 2 & 2 & 2 & 5 & huérfana \\
\hline 51 & 2 & 2 & 3 & 3 & 2 & 5 & 1 & 5 & 4 & 3 & 2 & 4 & huérfana \\
\hline 54 & 2 & 2 & 4 & 3 & 2 & 5 & 1 & 5 & 3 & 3 & 2 & 5 & 35 \\
\hline 55 & 2 & 2 & 4 & 3 & 2 & 6 & 1 & 3 & 3 & 3 & 2 & 2 & huérfana \\
\hline 56 & 1 & 2 & 4 & 3 & 2 & 8 & 1 & 5 & 3 & 3 & 2 & 6 & 180 \\
\hline 65 & 1 & 1 & 3 & 3 & 2 & 6 & 1 & 5 & 3 & 2 & 2 & 6 & huérfana \\
\hline 66 & 2 & 2 & 3 & 3 & 2 & 1 & 1 & 4 & 3 & 3 & 2 & 4 & huérfana \\
\hline 68 & 2 & 2 & 6 & 3 & 2 & 5 & 1 & 5 & 3 & 3 & 2 & 3 & 110 \\
\hline 71 & 2 & 2 & 4 & 3 & 2 & 5 & 1 & 5 & 3 & 3 & 2 & 4 & 34 \\
\hline 74 & 2 & 2 & 4 & 3 & 2 & 6 & 1 & 3 & 3 & 3 & 2 & 4 & 313 \\
\hline 75 & 2 & 2 & 3 & 4 & 2 & 5 & 1 & 5 & 3 & 3 & 2 & 2 & 157 \\
\hline 76 & 1 & 2 & 4 & 2 & 2 & 6 & 1 & 5 & 3 & 2 & 2 & 2 & 179 \\
\hline 77 & 2 & 2 & 2 & 3 & 2 & 1 & 1 & 5 & 3 & 3 & 2 & 5 & huérfana \\
\hline 78 & 2 & 2 & 4 & 3 & 2 & 5 & 1 & 4 & 3 & 3 & 2 & 4 & 117 \\
\hline 79 & 2 & 2 & 3 & 2 & 2 & 6 & 1 & 6 & 3 & 2 & 2 & 6 & huérfana \\
\hline 80 & 2 & 2 & 4 & 2 & 2 & 5 & 1 & 4 & 3 & 2 & 2 & 4 & huérfana \\
\hline 81 & 1 & 2 & 4 & 3 & 2 & 6 & 1 & 5 & 4 & 2 & 2 & 6 & huérfana \\
\hline 82 & 1 & 2 & 4 & 3 & 2 & 6 & 1 & 5 & 3 & 2 & 2 & 0 & 190 \\
\hline 83 & 1 & 2 & 4 & 3 & 2 & 6 & 1 & 5 & 3 & 2 & 2 & 0 & 190 \\
\hline 84 & 2 & 2 & 4 & 2 & 2 & 6 & 1 & 3 & 3 & 3 & 2 & 3 & huérfana \\
\hline 85 & 2 & 2 & 4 & 3 & 2 & 6 & 1 & 6 & 3 & 3 & 2 & 1 & 375 \\
\hline 86 & 1 & 2 & 4 & 3 & 2 & 6 & 1 & 5 & 3 & 2 & 2 & 0 & 190 \\
\hline 87 & 2 & 2 & 4 & 2 & 2 & 6 & 1 & 3 & 3 & 3 & 2 & 3 & huérfana \\
\hline 88 & 1 & 2 & 4 & 3 & 2 & 6 & 1 & 5 & 3 & 2 & 2 & 0 & 190 \\
\hline 89 & 2 & 2 & 3 & 3 & 2 & 5 & 1 & 5 & 3 & 3 & 2 & 4 & 125 \\
\hline 90 & 2 & 2 & 4 & 3 & 2 & 6 & 1 & 3 & 3 & 3 & 2 & 3 & 251 \\
\hline 91 & 2 & 2 & 4 & 3 & 2 & 5 & 1 & 4 & 3 & 2 & 2 & 4 & huérfana \\
\hline 92 & 1 & 2 & 4 & 3 & 2 & 6 & 1 & 5 & 3 & 2 & 2 & 6 & 151 \\
\hline 93 & 2 & 2 & 3 & 3 & 2 & 5 & 1 & 7 & 3 & 3 & 3 & 3 & 721 \\
\hline 94 & 2 & 2 & 4 & 3 & 2 & 6 & 1 & 3 & 3 & 3 & 2 & 3 & 251 \\
\hline 95 & 2 & 2 & 4 & 3 & 2 & 5 & 1 & 4 & 3 & 2 & 2 & 3 & 377 \\
\hline 96 & 2 & 2 & 3 & 3 & 2 & 5 & 1 & 7 & 3 & 3 & 3 & 3 & 721 \\
\hline 97 & 2 & 2 & 3 & 3 & 2 & 5 & 1 & 5 & 3 & 3 & 2 & 6 & huérfana \\
\hline 98 & 2 & 2 & 3 & 1 & 2 & 5 & 1 & 5 & 3 & 3 & 2 & 6 & 733 \\
\hline 100 & 2 & 2 & 4 & 2 & 2 & 6 & 1 & 3 & 3 & 0 & 2 & 3 & huérfana \\
\hline 103 & 2 & 2 & 3 & 2 & 1 & 5 & 1 & 4 & 3 & 2 & 2 & 4 & huérfana \\
\hline 105 & 2 & 2 & 4 & 3 & 2 & 5 & 1 & 6 & 1 & 2 & 2 & 3 & huérfana \\
\hline 106 & 2 & 2 & 4 & 3 & 2 & 5 & 1 & 7 & 3 & 5 & 3 & 3 & 229 \\
\hline
\end{tabular}

MIRU: micobacterial intersperse repetitive unit. VNTR: variable number tandem repeat. MIT: MIRU international type. *EI MIT para cada patrón de 12 dígitos fue obtenido en la base de datos del Instituto Pasteur-Guadeloupe (SITVITWEB). espoligotipos en la base de datos SITVITWEB, permitió obtener el número SIT en 19 de ellos, mientras que 6 patrones correspondieron a perfiles huérfanos (sin SIT).

Los cuatro SIT más frecuentes corresponden al SIT42 (ausencia del espaciador 21 al 24 y del 33 al 36), SIT33 (ausencia del espaciador 9 al 11,21 al 24 y 33 al 36), SIT53 (ausencia del espaciador desde 33 al 36), y SIT 52 (ausencia del espaciador 33 al 36 y 40), encontrados en $8,4,4$ y 3 cepas, respectivamente. También se encontró el SIT 1 ( $n=2$ cepas) que se caracteriza por la ausencia de los interespaciadores 1 al 34 en la región DR. Los resultados de espoligotipo y los SIT encontrados para cada cepa se muestran en la Tabla 3.

\section{Genotipos}

Para 86\% (37/43) de las cepas estudiadas fue asignado un genotipo previamente descrito en la literatura, mientras que al 14\% restante no se le asignó alguno, ("huérfanas"). Los resultados de la distribución de los genotipos y subgenotipos se muestran en la Tabla 4, donde se observa que el genotipo más frecuente fue el LAM $(39,5 \%)$, seguido por el genotipo $\mathrm{T}(32,5 \%)$, genotipo $\mathrm{H}(7,0 \%)$, genotipo Beijing $(4,7 \%)$ y genotipo $\mathrm{X}(2,3 \%)$.

\section{Discusión}

Este es el primer estudio realizado en Santiago de Chile que describe los genotipos del $\mathrm{cMtb}$, basado en dos metodologías complementarias (MIRU-VNTR y espoligotipificación). Se describe además el genotipo Beijing y se demuestra la predominancia del genotipo LAM y T. Estudios latinoamericanos más extensos realizados en Brasil, Argentina, Venezuela, Paraguay, Perú, Guayana-Francesa y Colombia muestran una clara predominancia del genotipo LAM en relación a los genotipos T y Haarlem ${ }^{1,6,22-27}$.

Nuestro estudio encuentra que ambos LAM y T son los más frecuentes en porcentajes relativamente similares (39,5 y $32,5 \%$, respectivamente); en cambio, el genotipo Haarlem, que en algunos países ocupa la segunda frecuencia (Argentina, Colombia, Perú y Paraguay), en este estudio corresponde a sólo $7 \%$ de las cepas halladas $6,24,26,27$.

El hallazgo en nuestro país de dos cepas del genotipo Beijing motiva a continuar el estudio ampliando el número de cepas y las zonas geográficas a estudiar. Lamentablemente, en el presente trabajo no disponemos de mayor información clínico/epidemiológica de los pacientes, como nacionalidad, co-infección por VIH u otra condición asociada a la enfermedad. Sin embargo, es necesario continuar estos estudios ya que el genotipo Beijing se ha asociado a mayor transmisibilidad, un curso de la enfermedad más agresivo y circulación de cepas de cMtb multi-resistente. 


\begin{tabular}{|c|c|c|c|c|c|c|c|c|c|c|c|c|c|c|c|c|c|c|c|c|c|c|c|c|c|c|c|c|c|c|c|c|c|c|c|c|c|c|c|}
\hline n aislamiento & \multicolumn{38}{|c|}{ Espoligotipo* } & \multirow{2}{*}{$\begin{array}{c}\text { SIT\# } \\
\text { huérfana }\end{array}$} \\
\hline 37 & 1 & 1 & 1 & 1 & 1 & 0 & 0 & 11 & 10 & 1 & 1 & 1 & 0 & 1 & 11 & 11 & 1 & 1 & 1 & 11 & 11 & 11 & 11 & 11 & 11 & 0 & 1 & 1 & 1 & 0 & 00 & 00 & 01 & 1 & 1 & 1 & 11 & 11 & \\
\hline 39 & 1 & 1 & 1 & 1 & 1 & 1 & 1 & 11 & 11 & 1 & 1 & 1 & 0 & 0 & 00 & 00 & 1 & 1 & 1 & 11 & 11 & 11 & 11 & 11 & 11 & 1 & 1 & 1 & 1 & 0 & 0 & 00 & 01 & 1 & 1 & 1 & 11 & 11 & 219 \\
\hline 42 & 1 & 1 & 1 & 1 & 1 & 1 & 1 & 11 & 11 & 1 & 1 & 1 & 1 & 1 & 11 & 11 & 1 & 1 & 0 & 00 & 00 & 01 & 11 & 11 & 11 & 1 & 1 & 1 & 1 & 0 & 00 & 00 & 01 & 1 & 1 & 1 & 11 & 11 & 42 \\
\hline 43 & 1 & 1 & 1 & 1 & 1 & 1 & 1 & 11 & 11 & 1 & 1 & 1 & 1 & 1 & 11 & 11 & 1 & 1 & 0 & 00 & 00 & 01 & 11 & 11 & 11 & 1 & 1 & 1 & 1 & 0 & 00 & 00 & 01 & 1 & 1 & 1 & 11 & 11 & 42 \\
\hline 47 & 1 & 1 & 1 & 1 & 1 & 1 & 1 & 11 & 11 & 1 & 1 & 1 & 1 & 1 & 11 & 11 & 1 & 1 & 1 & 11 & 11 & 11 & 11 & 11 & 11 & 1 & 1 & 1 & 1 & 0 & 00 & 00 & 01 & 1 & 1 & 1 & 11 & 11 & 53 \\
\hline 50 & 1 & 1 & 1 & 1 & 1 & 1 & 1 & 11 & 11 & 1 & 1 & 1 & 1 & 1 & 11 & 11 & 1 & 1 & 0 & 00 & 00 & 01 & 11 & 11 & 11 & 0 & 1 & 1 & 1 & 0 & 00 & 00 & 01 & 1 & 1 & 1 & 11 & 11 & 64 \\
\hline 51 & 1 & 1 & 1 & 1 & 1 & 1 & 1 & 11 & 11 & 1 & 1 & 1 & 1 & 1 & 11 & 11 & 1 & 1 & 1 & 11 & 11 & 11 & 11 & 11 & 11 & 1 & 1 & 1 & 1 & 0 & 00 & 00 & 01 & 1 & 1 & 1 & 11 & 11 & 53 \\
\hline 54 & 1 & 1 & 1 & 1 & 1 & 1 & 1 & 11 & 11 & 1 & 1 & 1 & 1 & 1 & 11 & 10 & 1 & 1 & 1 & 11 & 11 & 11 & 11 & 11 & 11 & 1 & 1 & 1 & 1 & 0 & 00 & 00 & 01 & 1 & 1 & 0 & 11 & 11 & 336 \\
\hline 55 & 1 & 1 & 1 & 1 & 1 & 1 & 1 & 10 & $\begin{array}{ll}0 & 0\end{array}$ & 0 & 1 & 1 & 1 & 1 & 11 & 11 & 1 & 1 & 0 & 00 & 00 & 01 & 11 & 11 & 11 & 1 & 1 & 1 & 1 & 0 & 00 & 00 & 01 & 1 & 1 & 1 & 11 & 11 & 33 \\
\hline 56 & 1 & 1 & 1 & 1 & 1 & 1 & 1 & 11 & 11 & 1 & 1 & 1 & 1 & 1 & 11 & 11 & 1 & 1 & 0 & 0 & 00 & $\begin{array}{ll}0 & 1\end{array}$ & 11 & 11 & 11 & 1 & 1 & 1 & 1 & 0 & 00 & 00 & 01 & 1 & 1 & 1 & 11 & 11 & 42 \\
\hline 65 & 1 & 1 & 1 & 1 & 1 & 1 & 1 & 11 & 11 & 1 & 1 & 1 & 1 & 1 & 11 & 11 & 1 & 1 & 0 & 00 & 00 & $\begin{array}{ll}0 & 1\end{array}$ & 11 & 11 & 11 & 1 & 1 & 1 & 1 & 0 & 00 & 00 & 01 & 1 & 1 & 1 & 11 & 11 & 42 \\
\hline 66 & 1 & 1 & 1 & 0 & 1 & 1 & 1 & 11 & 10 & 1 & 1 & 1 & 1 & 1 & 11 & 11 & 1 & 1 & 1 & 11 & 11 & 11 & 11 & 11 & 11 & 1 & 1 & 1 & 1 & 0 & 00 & 00 & 01 & 1 & 1 & 1 & 11 & 11 & 1877 \\
\hline 68 & 1 & 1 & 1 & 1 & 1 & 1 & 1 & 11 & 11 & 1 & 1 & 1 & 1 & 1 & 11 & 11 & 1 & 1 & 1 & 11 & 11 & 11 & 11 & 11 & 11 & 0 & 1 & 0 & 1 & 0 & 00 & 00 & 01 & 1 & 1 & 1 & 11 & 11 & 746 \\
\hline 71 & 1 & 1 & 1 & 1 & 1 & 1 & 1 & 11 & 11 & 1 & 1 & 1 & 1 & 1 & 11 & 11 & 1 & 1 & 1 & 11 & 11 & 11 & 11 & 11 & 11 & 1 & 1 & 1 & 1 & 0 & 0 & 00 & 01 & 1 & 1 & 0 & 11 & 11 & 52 \\
\hline 74 & 1 & 1 & 1 & 0 & 1 & 1 & 0 & 10 & $\begin{array}{ll}0 & 1\end{array}$ & 0 & 0 & 1 & 0 & 1 & 11 & 11 & 1 & 1 & 0 & 11 & 10 & 01 & 11 & 11 & 11 & 0 & 1 & 1 & 1 & 0 & 0 & 00 & 01 & 1 & 1 & 1 & 11 & 11 & huérfana \\
\hline 75 & 1 & 1 & 1 & 1 & 1 & 1 & 1 & 11 & 11 & 1 & 1 & 1 & 1 & 1 & 11 & 11 & 1 & 1 & 1 & 11 & 10 & $\begin{array}{ll}0 & 1\end{array}$ & 11 & 11 & 11 & 1 & 1 & 1 & 1 & 0 & 0 & 00 & 01 & 1 & 1 & 1 & 11 & 11 & 373 \\
\hline 76 & 1 & 1 & 1 & 1 & 1 & 1 & 1 & 11 & 11 & 1 & 1 & 1 & 1 & 1 & 10 & 00 & 0 & 0 & 0 & 11 & 11 & 11 & 11 & 11 & 11 & 0 & 1 & 1 & 1 & 0 & 00 & 00 & 01 & 1 & 1 & 1 & 11 & 11 & 22 \\
\hline 77 & 1 & 1 & 1 & 1 & 1 & 1 & 1 & 11 & 11 & 1 & 1 & 1 & 1 & 1 & 11 & 11 & 1 & 1 & 1 & 11 & 11 & 11 & 11 & 11 & 11 & 1 & 1 & 1 & 1 & 0 & 0 & 00 & 01 & 1 & 1 & 1 & 11 & 11 & 53 \\
\hline 78 & 1 & 1 & 1 & 1 & 1 & 1 & 1 & 11 & 11 & 1 & 1 & 1 & 1 & 1 & 11 & 11 & 0 & 1 & 1 & 10 & 00 & 01 & 11 & 11 & 11 & 1 & 1 & 1 & 1 & 0 & 00 & 00 & 01 & 0 & 0 & 1 & 11 & 11 & 39 \\
\hline 79 & 1 & 1 & 1 & 1 & 1 & 1 & 1 & 11 & 11 & 1 & 1 & 1 & 1 & 1 & 11 & 11 & 1 & 1 & 0 & 0 & 00 & 01 & 11 & 11 & 11 & 1 & 1 & 1 & 1 & 0 & 00 & 00 & 01 & 1 & 1 & 1 & 11 & 11 & 42 \\
\hline 80 & 1 & 1 & 1 & 1 & 1 & 0 & 0 & 11 & 11 & 1 & 1 & 1 & 1 & 1 & 11 & 11 & 1 & 1 & 1 & 11 & 11 & 11 & 11 & 11 & 11 & 0 & 1 & 1 & 1 & 0 & 00 & 00 & 01 & 1 & 1 & 1 & 11 & 11 & huérfana \\
\hline 81 & 1 & 1 & 1 & 1 & 1 & 1 & 1 & 11 & 11 & 1 & 1 & 1 & 1 & 1 & 11 & 11 & 1 & 1 & 0 & 0 & 00 & $\begin{array}{ll}0 & 1\end{array}$ & 11 & 10 & 00 & 1 & 1 & 1 & 1 & 0 & 00 & 00 & 01 & 1 & 1 & 1 & 11 & 11 & 452 \\
\hline 82 & 1 & 1 & 1 & 1 & 1 & 1 & 1 & 11 & 11 & 1 & 1 & 1 & 1 & 1 & 11 & 11 & 1 & 1 & 0 & 00 & 00 & $\begin{array}{ll}0 & 1\end{array}$ & 11 & 11 & 11 & 1 & 1 & 1 & 1 & 0 & 00 & 00 & 01 & 1 & 1 & 1 & 11 & 11 & 42 \\
\hline 83 & 1 & 1 & 1 & 1 & 1 & 0 & 1 & 11 & 11 & 1 & 1 & 1 & 1 & 1 & 11 & 11 & 1 & 1 & 0 & 11 & 10 & 01 & 11 & 11 & 11 & 1 & 1 & 1 & 1 & 0 & 0 & 00 & 01 & 1 & 1 & 1 & 11 & 11 & huérfana \\
\hline 84 & 1 & 1 & 1 & 1 & 1 & 1 & 1 & 10 & $\begin{array}{ll}0 & 0\end{array}$ & 0 & 1 & 1 & 1 & 1 & 11 & 11 & 1 & 1 & 0 & 00 & 00 & $\begin{array}{ll}0 & 1\end{array}$ & 11 & 11 & 11 & 1 & 1 & 1 & 1 & 0 & 00 & 00 & 01 & 1 & 1 & 1 & 11 & 11 & 33 \\
\hline 85 & 1 & 1 & 0 & 1 & 1 & 1 & 1 & 11 & 11 & 1 & 1 & 1 & 1 & 1 & 11 & 11 & 1 & 1 & 0 & 01 & 10 & $\begin{array}{ll}0 & 1\end{array}$ & 11 & 11 & 11 & 1 & 1 & 1 & 1 & 0 & 0 & 00 & 01 & 1 & 1 & 1 & 11 & 11 & 1474 \\
\hline 86 & 1 & 1 & 1 & 1 & 1 & 1 & 1 & 11 & 11 & 1 & 1 & 1 & 1 & 1 & 11 & 11 & 1 & 1 & 0 & 00 & 00 & $\begin{array}{ll}0 & 1\end{array}$ & 11 & 11 & 11 & 1 & 1 & 1 & 1 & 0 & 0 & 00 & 01 & 1 & 1 & 1 & 11 & 11 & 42 \\
\hline 87 & 1 & 1 & 1 & 1 & 1 & 1 & 1 & 10 & 00 & 0 & 1 & 0 & 1 & 1 & 11 & 11 & 1 & 1 & 0 & 00 & 00 & $\begin{array}{ll}0 & 1\end{array}$ & 11 & 11 & 11 & 1 & 1 & 1 & 1 & 0 & 00 & 00 & $\begin{array}{ll}0 & 1\end{array}$ & 1 & 1 & 1 & 11 & 11 & 211 \\
\hline 88 & 1 & 1 & 1 & 1 & 1 & 1 & 1 & 11 & 11 & 1 & 1 & 1 & 1 & 1 & 11 & 11 & 1 & 1 & 0 & 00 & 00 & $\begin{array}{ll}0 & 1\end{array}$ & 11 & 11 & 11 & 1 & 1 & 1 & 1 & 0 & 0 & 00 & 01 & 1 & 1 & 1 & 11 & 11 & 42 \\
\hline 89 & 1 & 1 & 1 & 1 & 1 & 1 & 1 & 11 & 11 & 1 & 1 & 1 & 1 & 1 & 11 & 11 & 1 & 1 & 1 & 11 & 11 & 11 & 11 & 11 & 11 & 1 & 1 & 1 & 1 & 0 & 00 & 00 & 01 & 1 & 1 & 1 & 11 & 11 & 53 \\
\hline 90 & 1 & 1 & 1 & 1 & 1 & 1 & 1 & 10 & $\begin{array}{ll}0 & 0\end{array}$ & 0 & 1 & 0 & 1 & 1 & 11 & 11 & 1 & 1 & 0 & 00 & 00 & $\begin{array}{ll}0 & 1\end{array}$ & 11 & 11 & 11 & 1 & 1 & 1 & 1 & 0 & 00 & 00 & 01 & 1 & 1 & 1 & 11 & 11 & 211 \\
\hline 91 & 1 & 1 & 1 & 1 & 1 & 1 & 1 & 11 & 11 & 1 & 1 & 1 & 1 & 1 & 11 & 11 & 0 & 1 & 1 & 10 & 00 & $\begin{array}{ll}0 & 1\end{array}$ & 11 & 11 & 11 & 1 & 1 & 1 & 1 & 0 & 00 & 00 & 01 & 0 & 0 & 1 & 11 & 11 & 39 \\
\hline 92 & 1 & 1 & 1 & 1 & 1 & 1 & 1 & 11 & 11 & 1 & 1 & 1 & 1 & 1 & 11 & 11 & 1 & 1 & 0 & 0 & 00 & $\begin{array}{ll}0 & 1\end{array}$ & 11 & 11 & 11 & 1 & 1 & 1 & 1 & 0 & 00 & 00 & 01 & 1 & 1 & 1 & 10 & 00 & 738 \\
\hline 93 & 0 & 0 & 0 & 0 & 0 & 0 & 0 & 00 & 00 & 0 & 0 & 0 & 0 & 0 & 00 & 00 & 0 & 0 & 0 & 00 & 00 & 00 & 00 & 00 & 00 & 0 & 0 & 0 & 0 & 0 & 0 & 11 & 11 & 1 & 1 & 1 & 11 & 11 & 1 \\
\hline 94 & 1 & 1 & 1 & 1 & 1 & 1 & 1 & 10 & $\begin{array}{ll}0 & 0\end{array}$ & 0 & 1 & 1 & 1 & 1 & 11 & 11 & 1 & 1 & 0 & 0 & 00 & $\begin{array}{ll}0 & 1\end{array}$ & 11 & 11 & 11 & 1 & 1 & 1 & 1 & 0 & 00 & 00 & 01 & 1 & 1 & 1 & 11 & 11 & 33 \\
\hline 95 & 1 & 1 & 1 & 1 & 1 & 1 & 1 & 1 & 11 & 1 & 1 & 1 & 1 & 1 & 11 & 11 & 1 & 1 & 1 & 11 & 11 & 11 & 11 & 11 & 11 & 1 & 0 & 0 & 0 & 0 & 00 & 00 & 01 & 0 & 0 & 1 & 11 & 11 & huérfana \\
\hline 96 & 1 & 1 & 1 & 1 & 1 & 0 & 0 & 11 & 10 & 1 & 1 & 1 & 1 & 1 & 11 & 11 & 0 & 1 & 1 & 10 & 00 & 01 & 11 & 11 & 11 & 1 & 1 & 1 & 1 & 0 & 00 & 00 & 01 & 0 & 0 & 1 & 1 & 11 & huérfana \\
\hline 97 & 1 & 1 & 1 & 1 & 1 & 1 & 1 & 11 & 11 & 1 & 1 & 1 & 1 & 1 & 11 & 11 & 1 & 1 & 1 & 11 & 11 & 11 & 11 & 11 & 11 & 1 & 1 & 1 & 1 & 0 & 00 & 00 & 01 & 1 & 1 & 0 & 11 & 11 & 52 \\
\hline 98 & 1 & 1 & 1 & 1 & 1 & 1 & 1 & 1 & 11 & 1 & 1 & 1 & 1 & 1 & 11 & 11 & 1 & 1 & 1 & 11 & 11 & 11 & 11 & 11 & 11 & 1 & 1 & 1 & 1 & 0 & 0 & 00 & 01 & 1 & 1 & 0 & 11 & 11 & 52 \\
\hline 100 & 1 & 1 & 1 & 1 & 1 & 1 & 1 & $1 \mathrm{c}$ & 00 & 0 & 1 & 1 & 1 & 1 & 11 & 11 & 1 & 1 & 0 & 0 & 00 & $\begin{array}{ll}0 & 1\end{array}$ & 11 & 11 & 11 & 1 & 1 & 1 & 1 & 0 & 0 & 00 & 01 & 1 & 1 & 1 & 11 & 11 & 33 \\
\hline 103 & 1 & 1 & 0 & 1 & 1 & 1 & 1 & 11 & 11 & 1 & 1 & 1 & 1 & 1 & 11 & 11 & 1 & 1 & 1 & 11 & 11 & 11 & 11 & 11 & 11 & 1 & 1 & 0 & 1 & 0 & 00 & 00 & 01 & 1 & 1 & 1 & 1 & 1 & 180 \\
\hline 105 & 1 & 1 & 1 & 1 & 1 & 1 & 1 & 11 & 11 & 1 & 1 & 1 & 1 & 1 & 11 & 11 & 1 & 1 & 1 & 11 & 10 & 00 & 00 & 00 & 00 & 0 & 0 & 0 & 1 & 0 & 0 & 00 & 01 & 1 & 0 & 0 & 01 & 11 & 948 \\
\hline 106 & 0 & 0 & 0 & 0 & 0 & 0 & & 00 & $\begin{array}{ll}0 & 0\end{array}$ & 0 & 0 & 0 & 0 & 0 & 00 & $\begin{array}{ll}0 & 0\end{array}$ & 0 & 0 & 0 & 00 & 00 & $\begin{array}{ll}0 & 0\end{array}$ & 00 & 00 & 00 & 0 & 0 & 0 & 0 & 0 & 01 & 11 & 11 & 1 & 1 & 1 & 11 & 11 & 1 \\
\hline
\end{tabular}

Finalmente, es llamativo que 14\% de las cepas estudiadas correspondieron a cepas no previamente clasificadas (cepas huérfanas), siendo esta proporción solamente superada por estudios realizados en Brasil ${ }^{22}$. Será de interés en estudios futuros con un mayor número de cepas corroborar si se mantiene esta proporción y determinar si alguna de estas pudiese corresponder a un clon "chileno" no previamente descrito.

\begin{tabular}{|c|c|c|}
\hline n de cepas (\%) & Genotipo & Subgenotipos \\
\hline $17(39,5)$ & LAM & $\operatorname{LAM9}(n=10), \operatorname{LAM} 3(n=6), \operatorname{LAM} 6(n=1)$ \\
\hline $14(32,5)$ & $\mathrm{T}$ & T1 $(n=9)$, T2 $(n=3)$, T4-CEU1 $(n=2)$ \\
\hline $3(7,0)$ & $\mathrm{H}$ & $\mathrm{H} 3(\mathrm{n}=3)$ \\
\hline $2(4,7)$ & Beijing & - \\
\hline $1(2,3)$ & x & $X 1(n=1)$ \\
\hline
\end{tabular}


El presente estudio destaca la importancia de conocer los genotipos predominantes en Chile, como varían en el tiempo y determinar la dinámica de la transmisión. La creciente inmigración, con nuevas cepas de $M$. tuberculosis, supone un reto de vigilancia epidemiológica en las que los estudios moleculares jugarán un rol importante en el control y contención de la enfermedad.

\section{Resumen}

Introducción: La genotipificación del complejo Mycobacterium tuberculosis (cMtbc) permite conocer los genotipos geográficamente predominantes. Algunos genotipos se diseminan con mayor rapidez y se asocian a multi-resistencia, tal como Beijing, reportado en América Latina en Perú. Existe poca información al respecto en Chile, sin reportes de la presencia de Beijing. Objetivo: Conocer los genotipos prevalentes en dos centros de salud de la Región Metropolitana de Chile con énfasis en la búsqueda de Beijing. Métodos: Se utilizaron dos métodos moleculares complementarios basados en la variación de las regiones de repeticiones directa en el genoma de M. tuberculosis (espoligotipificación) y número variable de repeticiones en tandem de las unidades repetitivas de interespaciadores micobacterianos (MIRU-VNTRs) y posterior análisis en bases de datos internacionales. Resultados: Se asignó un genotipo conocido a 37 de las 43 cepas estudiadas (86\%), mientras que en $14 \%$ no se asignó alguno. Los genotipos LAM y T fueron los más frecuentes $(39,5$ y $32,5 \%$, respectivamente), seguidos por Haarlem $(7,0 \%)$, Beijing (4,7\%) y X (2,3\%). Conclusión: Se describe por primera vez en Chile la presencia del genotipo Beijing en cepas de cMtb. Es necesario realizar una vigilancia epidemiológica molecular en el cMtb para conocer la dinámica de la transmisión en nuestro país.

\section{Referencias bibliográficas}

1.- Brudey K, Driscoll J R, Rigouts L, Prodinger W M, Gori A, Al-Hajoj S A, et al. Mycobacterium tuberculosis complex genetic diversity: mining the fourth international spoligotyping database (SpolDB4) for classification, population genetics and epidemiology. BMC Microbiol 2006; 6: 23.

2.- Pareek M, Evans J, Innes J, Smith G, Hingley-Wilson S, Lougheed K E, et al. Ethnicity and mycobacterial lineage as determinants of tuberculosis disease phenotype. Thorax 2013; 68 (3): 221-9.

3.- Gagneux S, DeRiemer K, Van T, Kato-Maeda M, de Jong BC, Narayanan S, et al. Variable host-pathogen compatibility in Mycobacterium tuberculosis. Proc Natl Acad Sci USA 2006; 103 (8): 2869-73.

4.- Kremer K, van Soolingen D, Frothingham R, Haas W H, Hermans P W, Martin C, et al. Comparison of methods based on different molecular epidemiological markers for typing of Mycobacterium tuberculosis complex strains: interlaboratory study of discriminatory power and reproducibility. J Clin Microbiol 1999; 37 (8): 2607-18.

5.- Ritacco V, Iglesias M J, Ferrazoli L, Monteserin J, Dalla Costa E R, Cebollada A, et al. Conspicuous multidrug-resistant Mycobacterium tuberculosis cluster strains do not trespass country borders in Latin America and Spain. Infect Genet Evol 2012; 12 (4): 711-7.

6.- Taype C A, Agapito J C, Accinelli R A, Espinoza J R, Godreuil S, Goodman S J, et al. Genetic diversity, population structure and drug resistance of Mycobacterium tuberculosis in
Peru. Infect Genet Evol 2012; 12 (3): 577-85.

7.- Kallenius G, Koivula T, Ghebremichael S, Hoffner S E, Norberg R, Svensson E, et al. Evolution and clonal traits of Mycobacterium tuberculosis complex in Guinea-Bissau. J Clin Microbiol 1999; 37 (12): 3872-8.

8.- Iwamoto T, Grandjean L, Arikawa K, Nakanishi N, Caviedes L, Coronel J, et al. Genetic diversity and transmission characteristics of Beijing family strains of Mycobacterium tuberculosis in Peru. PLoS One 2012; 7 (11): e49651.

9.- Mokrousov I, Ly H M, Otten T, Lan N N, Vyshnevskyi B, Hoffner S, et al. Origin and primary dispersal of the Mycobacterium tuberculosis Beijing genotype: clues from human phylogeography. Genome Res 2005; 15 (10): 1357-64.

10.- Von Groll A, Martin A, Félix C, Prata P F, Honscha G, Portaels F, et al. Fitness study of the RDRio lineage and Latin AmericanMediterranean family of Mycobacterium tuberculosis in the city of Rio Grande, Brazil. FEMS Immunol Med Microbiol 2010; 58 (1): 119-27.

11.- Mathema B, Kurepina N, Yang G, Shashkina E, Manca C, Mehaffy C, et al. Epidemiologic consequences of microvariation in Mycobacterium tuberculosis. J Infect Dis 2012; 205 (6): 964-74.

12.- Plikaytis B B, Marden J L, Crawford J T, Woodley C L, Butler W R, Shinnick T M. Multiplex PCR assay specific for the multidrug-resistant strain $\mathrm{W}$ of Mycobacterium tuberculosis. J Clin Microbiol 1994; 32 (6): 1542-6.

13.- Ritacco V, López B, Cafrune P I, Ferrazoli L, Suffys P N, Candia N, et al. Mycobacterium tuberculosis strains of the Beijing genotype are rarely observed in tuberculosis patients in South America. Mem Inst Oswaldo Cruz 2008; 103 (5): 489-92.

14.- Mancilla E M, Martínez H A, Palavecino B C, Rehren S G, Lucero L P, Leon R G, et al. Genetic variants of Mycobacterium tuberculosis isolated from patients of the Xth Region of Chile. Rev Chilena Infectol 2006; 23 (3): 220-5.

15.- Kasai H, Ezaki T, Harayama S. Differentiation of phylogenetically related slowly growing mycobacteria by their gyrB sequences. J Clin Microbiol 2000; 38 (1): 301-8.

16.- Cowan L S, Mosher L, Diem L, Massey J P, Crawford J T. Variable-number tandem repeat typing of Mycobacterium tuberculosis isolates with low copy numbers of IS6110 by using mycobacterial interspersed repetitive units. $\mathrm{J}$ Clin Microbiol 2002; 40 (5): 1592-602.

17.- Kamerbeek J, Schouls L, Kolk A, van Agterveld M, van Soolingen D, Kuijper S, et al. Simultaneous detection and strain differentiation of Mycobacterium tuberculosis for diagnosis and epidemiology. J Clin Microbiol 1997; 35 (4): 907-14.

18.- Abadia E, Zhang J, Ritacco V, Kremer $\mathrm{K}$, Ruimy R, Rigouts L, et al. The use of microbead-based spoligotyping for Mycobacterium tuberculosis complex to evaluate the quality of the conventional method: providing guidelines for Quality Assurance when working on membranes. BMC Infect Dis 2011; 11: 110.

19.- Demay C, Liens B, Burguiere T, Hill V, Couvin $\mathrm{D}$, Millet J, et al. SITVITWEB-a publicly available international multimarker database for studying Mycobacterium tuberculosis genetic diversity and molecular epidemiology. Infect 
Genet Evol 2012; 12 (4): 755-66.

20.- Shabbeer A, Ozcaglar C, Yener B, Bennett K P. Web tools for molecular epidemiology of tuberculosis. Infect Genet Evol 2012; 12 (4): 767-81.

21.- Weniger T, Krawczyk J, Supply P, Niemann S, Harmsen D. MIRU-VNTRplus: a web tool for polyphasic genotyping of Mycobacterium tuberculosis complex bacteria. Nucleic Acids Res 2010; 38 (Web Server issue): W326-31.

22.- Gomes H M, Elias A R, Oelemann M A, Pereira M A, Montes F F, Marsico A G, et al. Spoligotypes of Mycobacterium tuberculosis complex isolates from patients residents of 11 states of Brazil. Infect Genet Evol 2012; 12 (4): 649-56.

23.- Gonzalo X, Ambroggi M, Cordova E, Brown T, Poggi S, Drobniewski F. Molecular epidemiology of Mycobacterium tuberculosis, Buenos Aires, Argentina. Emerg Infect Dis 2011; 17 (3): 528-31.

24.- Ritacco V, López B, Ambroggi M, Palmero D, Salvadores B, Gravina E, et al. HIV infection and geographically bound transmission of drugresistant tuberculosis, Argentina. Emerg Infect Dis 2012; 18 (11): 1802-10.

25.- Aristimuno L, Armengol R, Cebollada A, Espana M, Guilarte A, Lafoz C, et al. Molecular characterisation of Mycobacterium tuberculosis isolates in the First National Survey of Antituberculosis Drug Resistance from Venezuela. BMC Microbiol 2006; 6: 90.

26.- Candia N, López B, Zozio T, Carrivale M, Díaz C, Russomando G, et al. First insight into Mycobacterium tuberculosis genetic diversity in Paraguay. BMC Microbiol 2007; 7: 75.

27.- Cerezo I, Jiménez Y, Hernández J, Zozio T, Murcia M I, Rastogi N. A first insight on the population structure of Mycobacterium tuberculosis complex as studied by spoligotyping and MIRU-VNTRs in Bogota, Colombia. Infect Genet Evol 2012; 12 (4): 657-63. 